\title{
Publisher Correction: Colchicine acts selectively in the liver to induce hepatokines that inhibit myeloid cell activation
}

Jui-Hsia Weng (1), Peter David Koch, Harding H. Luan, Ho-Chou Tu, Kenichi Shimada (D), Iris Ngan, Richard Ventura, Ruomu Jiang and Timothy J. Mitchison (1)

Correction to: Nature Metabolism https://doi.org/10.1038/s42255-021-00366-y, published online 12 April 2021.

In the version of this article initially published, affiliations 3 and 4 were switched; the correct affiliations are ${ }^{3} \mathrm{NGM}$ Biopharmaceuticals, South San Francisco, CA, USA and ${ }^{4}$ Alnylam Pharmaceuticals, Inc., Cambridge, MA, USA. In addition, the colors in Fig. 5k were rendered in greyscale in the published version. Finally, a middle initial has been added for author Harding H. Luan. The errors have been corrected in the HTML and PDF versions of the article.

Published online: 5 May 2021

https://doi.org/10.1038/s42255-021-00397-5

(C) The Author(s), under exclusive licence to Springer Nature Limited 2021 